\title{
The correct application of Poynting's theorem to the time-dependent magnetosphere: reply to Heikkila
}

\author{
M. Lockwood, C. J. Davis \\ Rutherford Appleton Laboratory, Chilton, Didcot, Oxfordshire, OX11 0QX, UK
}

Received: 28 February 1997 / Revised: 26 September 1997 / Accepted: 23 March 1998

In his comment, Heikkila (1998) raises a number of objections to the concept of time-varying magnetic reconnection, and magnetic reconnection in general, which was obviously the basis of our paper on the longitudinal extent of magnetopause reconnection pulses (Lockwood and Davis, 1996). These objections are invalid.

Much of the problem arises because Heikkila relies heavily on a simple electrical circuit analogy for the magnetosphere. As an analogy, this can be useful but it remains only an analogy. Thus, for example, Heikkila continues to inappropriately and erroneously invoke Lenz's law. However, the most serious effect in his comment is on how he applies Poynting's theorem. He refers to the reconnecting dayside magnetopause as a load (valid in the sense that $\mathbf{J} \cdot \mathbf{E}>0$ ) and his analogy demands that this be connected to a dynamo (by which he means a region in which $\mathbf{J} \cdot \mathbf{E}<0$ ) in a current loop. This can be valuable for the steady-state magnetosphere, with allowance for the fact that there are a number of complex current loops (Cowley, 1991). However, the rise and fall of magnetic energy density must be added in the non-steady situation we discuss. Because Heikkila does not do this, his simple load-and-dynamo analogy is invalid and inappropriate, as are his objections to our paper.

Let us briefly derive and apply Poynting's theorem. If we compress a magnetic field $B$ by $\mathrm{d} \tau$ in volume, we do work against the Maxwell magnetic pressure of $\partial W_{\mathrm{B}}=\left(B^{2} / 2 \mu_{\mathrm{o}}\right) \mathrm{d} \tau$. Thus the rate at which energy is stored in the magnetic field in a volume $\tau$ is:

$\partial W_{\mathrm{B}} / \partial t=\partial / \partial t\left(\int_{\tau}\left(B^{2} / \mu_{\mathrm{o}}\right) \mathrm{d} \tau\right)=\left(1 / 2 \mu_{\mathrm{o}}\right) \int_{\tau}(\mathbf{B} \cdot \partial \mathbf{B} / \partial t) \mathrm{d} \tau$

If we substitute from Faraday's law, use the vector relation $\nabla \cdot(\mathbf{E} \times \mathbf{B})=\mathbf{B} \cdot(\nabla \times \mathbf{E})-\mathbf{E} \cdot(\nabla \times \mathbf{B})$, Amp-

Correspondence to: M. Lockwood e-mail: mike@eiscat.ag.rl.ac.uk ère's law, and the definition of Poynting flux $(\mathbf{S}=(\mathbf{E} \times \mathbf{B}) / \mu)$, we arrive at Poynting's theorem for a plasma:

$$
\int_{\tau} \mathbf{J} \cdot \mathbf{E} \mathrm{d} \tau=-\partial W_{\mathrm{B}} / \partial t-\int_{A} \mathbf{S} \cdot d s
$$

where the surface $A$ surrounds the volume $\tau$. The second term on the right is the divergence of the Poynting flux and the term on the left the ohmic heating term. Notice that we have used only fundamental laws, i.e. Maxwell's equations for a low-Q (the displacement current having been neglected when we applied Ampère's law), nonmagnetized $\left(\mu_{\mathrm{r}}=1, \mu=\mu_{\mathrm{o}}\right)$ medium. A more general formulation of Poynting's theorem is obtained by adding the electric energy density $W_{\mathrm{E}}=\epsilon_{\mathrm{o}} \mathrm{E}^{2} / 2$ to the magnetic energy density $W_{\mathrm{B}}$ in Eq. (1) and was given by Heikkila. We can neglect $W_{\mathrm{E}}$ because it is much smaller than $W_{\mathrm{B}}$ at non-relativistic speeds. In fact, it did not arise in Eq. (1) because we neglected the displacement current $(\mathrm{Q}<<1)$ in our derivation. Neglecting $W_{\mathrm{E}}$ is of no consequence, but it does make understanding energy flow in the magnetosphere easier.

If we consider steady state, $\partial W_{\mathrm{B}} / \partial \mathrm{t}$ is zero and Eq. (1) shows that a region where $\mathbf{J} \cdot \mathbf{E}>0$ is a sink of Poynting flux. Conversely regions of $\mathbf{J} \cdot \mathbf{E}<0$ are sources of $S$. The current systems contributing to the local $J$ can be complex and there are no general restrictions on where the source or sources of any Poynting flux entering the volume $\tau$ are located. Nor are there any restrictions on where any $S$ leaving the volume $\tau$ must be deposited.

On the other hand, the non-steady situations we address in our paper, $\partial W_{\mathrm{B}} / \partial \mathrm{t}$ is not zero. By insisting that every region of $\mathbf{J} \cdot \mathbf{E}>0$ there must be a corresponding region of $\mathbf{J} \cdot \mathbf{E}<0$, Heikkila is neglecting this term. One possibility is that there is no divergence of $S$, in which case Eq. (1) yields $\int \mathbf{J} \cdot \mathbf{E} \mathrm{d} \tau=-\partial W_{\mathrm{B}} / \partial \mathrm{t}$, i.e. the energy being dissipated in the "load" by ohmic heating is equal to the simultaneous local loss of magnetic energy density. Another possibility is that the 
energy dissipated corresponds to a local steady-state sink of Poynting flux, and this $\mathbf{S}$ arises from a source (or sources) where $\mathbf{J} \cdot \mathbf{E}=0$ and where there is a loss of magnetic energy density $\left(\int_{\mathrm{A}} \mathbf{S} \cdot d s=-\partial W_{\mathrm{B}} / \partial \mathrm{t}\right)$. In both these two examples, we have a region of $\mathbf{J} \cdot \mathbf{E}>0$ without a corresponding region of $\mathbf{J} \cdot \mathbf{E}<0$ (instead, there are regions where $W_{\mathrm{B}}$ is decreasing). Heikkila's assertion that there must always be a dynamo and a load is incorrect. Notice also that sources of $S$ are not necessarily on the same current loops as the sinks. Heikkila dismisses this "for causal reasons", but to do so is to deny the existence mutual inductance phenomena (Heikkila's simple load-and-dynamo circuit analogy does not include any transformers, let alone inductors or capacitors).

Let us look at two non-steady situations in the magnetosphere. The dashed lines in Fig. 1 show the Poynting flux for southward IMF giving steady magnetopause reconnection at $\mathrm{X}$. This figure is based on that by Cowley (1991), but is adapted to a non-steady situation of a substorm growth phase. At the dayside

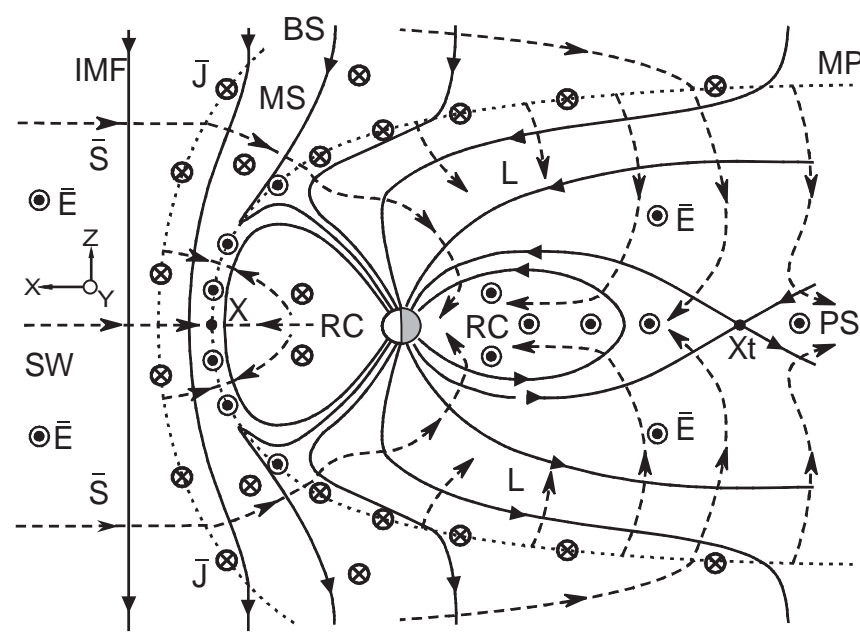

Fig. 1. Poynting's theorem applied to the non-steady case of the magnetosphere during a substorm growth phase. A noon-midnight schematic cross-section of the magnetosphere is presented viewed from dusk with the sun to the left. When the IMF is southward $\left(B_{\mathrm{z}}<0\right)$, field lines (solid lines) are opened at the magnetopause reconnection site, $X$, and convect antisunward under the action of the solar wind electric field, E, which exists in the Earth's frame of reference and everywhere points out of the plane of the diagram in the $+\mathrm{Y}$ direction. The flow of energy, or Poynting flux, $\mathbf{S}=(\mathbf{E} \times \mathbf{B}) / \mu_{\mathrm{o}}$, is shown by the dashed lines. Unless otherwise marked as electric field $\mathbf{E}$, vectors into and out of the plane of the diagram are currents, $\mathbf{J}$. The open field lines are generated at $X$ and closed again (at a slower rate) at $X \mathrm{t}$. Labelled regions and boundaries are: solar wind SW; interplanetary magnetic field IMF; bow shock BS; magnetopause MP; magnetosheath MS; tail lobe L; the plasma sheet PS and the ring current RC. The dayside magnetopause (Chapman-Ferraro) currents are in the $+\mathrm{Y}$ direction, making $\mathbf{J} \cdot \mathbf{E}>0$, i.e. there is a sink of $S$. At latitudes above the magnetic cusp, the currents are into the diagram, making $\mathbf{J} \cdot \mathbf{E}<0$, i.e. a source of $\mathbf{S}$. In the substorm growth phase, the energy extracted from the solar wind at the tail magnetopause is stored as magnetic energy in the tail lobe because the lobe field and energy density increase as open magnetic flux accumulates. In addition, some is deposited in the plasma sheet, ring current and the ionosphere because some reconnection is taking place at $X \mathrm{t}$ (Adapted from Cowley, 1991) magnetopause (MP) $\mathbf{J} \cdot \mathbf{E}>0$ and this is a sink of $\mathbf{S}$ which originates from the solar wind (SW), the bow shock (BS) and the dayside ring current (RC). On the other hand, the tail lobe boundary magnetopause, antisunward of the cusps, is a region where $\mathbf{J} \cdot \mathbf{E}<0$ and is a source of Poynting flux. Because tail reconnection is ongoing at $\mathrm{Xt}$, some of this Poynting flux is deposited as ohmic heating on the cross-tail current of the plasma sheet (PS), in the nightside ring current (RC) and in the ionosphere where $\mathbf{J} \cdot \mathbf{E}>0$. However, because the reconnection voltage at $\mathrm{Xt}$ is lower than that at $\mathrm{X}, B$ and therefore $W_{\mathrm{B}}$ are increasing in the tail lobes (L), which are therefore sinks of $S$ in this growth phase situation. (Of course later, during the expansion and recovery phases of the substorm cycle, $B$ and $W_{\mathrm{B}}$ will decrease in the tail lobes as reconnection at $\mathrm{Xt}$ dominates - the tail lobes then act as sources of $S$, which is deposited in the plasma sheet, the ring current and the ionosphere).

This first example demonstrates the importance of considering the $\partial W_{\mathrm{B}} / \partial \mathrm{t}$ term when applying Poynting's theorem to the non-steady magnetosphere. Applying this to the specific case of pulsed magnetopause reconnection, we will now show that we did not make what Heikkila terms "a serious error in logic".

Figure 2 shows the situation shortly after the onset of a pulse of magnetopause reconnection between the magnetosheath field $B_{\mathrm{sh}}$ and the magnetospheric field $B_{\mathrm{sp}}$. The shaded region is the region to which information about the onset of reconnection has reached. Outside this region there is no change to any of the physical properties of the plasma. The topology of the

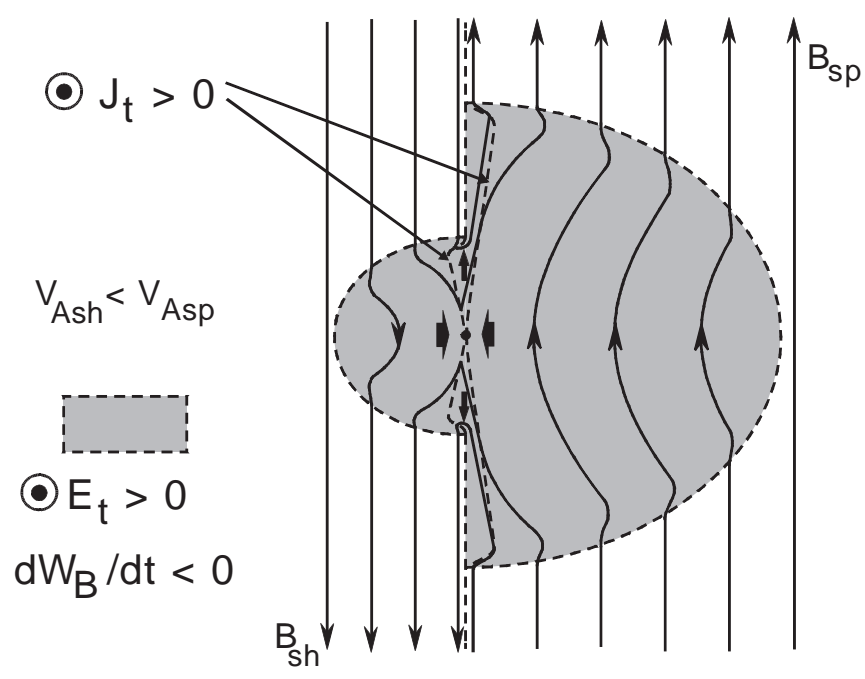

Fig. 2. Schematic of the dayside magnetopause shortly after the onset of a pulse of reconnection between the magnetosheath and magnetospheric fields $B_{\mathrm{sh}}$ and $B_{\mathrm{sp}}$. Information about the onset has spread into the shaded region, in which the tangential electric field causes inflow towards the current sheet and the magnetic energy density is decreased. The shaded region is therefore a source of Poynting flux, which is directed towards the current sheet on both sides. This is dissipated in Ohmic heating by the currents that flow in the two rotational discontinuities emanating from the reconnection site (dashed lines). Dissipated energy does not come from outside the shaded region, which expands with time 
reconnected field lines has changed from closed to open but information on this change has yet to reach outside the shaded area. The Alfvén speed and the sound speed are greater on the magnetospheric side of the boundary and so the change has propagated further away from the reconnection site into the magnetosphere than it has into the magnetosheath. Within the shaded region, the tangential electric field (the reconnection rate, $E_{\mathrm{t}}$ ) acts out of the plane of the diagram and corresponds to the plasma inflow toward the current sheet and the outflow along the current sheet. Current $J_{\mathrm{t}}$ flows out of the plane of the diagram in the two rotational discontinuities emanating from the reconnection site (dashed lines) such that $\mathbf{J}_{\mathrm{t}} \cdot \mathbf{E}_{\mathrm{t}}>0$. (This energy is mainly manifested as the accelerated ion flows along the boundary). The electric field $\mathbf{E}_{\mathrm{t}}$ gives Poynting flux $\mathbf{S}=(\mathbf{E} \times \mathbf{B}) / \mu_{\mathrm{o}}$ in the shaded regions which is towards this ohmic dissipation, on both sides of the boundary. The source of this Poynting flux is the reduction of magnetic field in the shaded region where $\partial W_{\mathrm{B}} / \partial \mathrm{t}<0$. Therefore the source of the energy deposited in the early stages of the reconnection pulse is close to the sink (within the sphere of influence). Therefore we were not invoking sources of S outside of the "sphere of influence" as Heikkila claims we do.

Heikkila's neglect of the $\partial W_{\mathrm{B}} / \partial \mathrm{t}$ term is the main reason that he makes erroneous objections to our paper. However, his comment is also filled with a large number of misleading, incorrect, irrelevant and unsupported statements. So that these do not remain unchallenged to corrupt the publication record, we quickly note just some of them below.

In the abstract and his conclusion, Heikkila claims "Lockwood and Davis use the wrong contour in their attempt to evaluate the emf". This does not make sense. We apply Faraday's law to a loop ABba. Faraday's law is a universal law of nature and must apply to any fixed loop and so there can be no "right" or "wrong" loop to apply it to. We chose the loop ABba because we wanted to learn about the voltage along the merging gap ab and the reconnection voltage which, by definition, is applied to $\mathrm{AB}$.

Heikkila's choice of the data shown by Woch and Lundin (1992) as an example of cusp ion steps is very misleading. We did not cite this paper as an example of cusp ion steps. Woch and Lundin showed very interesting "overlapping injection signatures" but these are not what we referred to as "cusp ion steps". There are a host of observations of cusp ion steps without the complication of overlapping (many were cited in our original paper and others have recently been reported (e.g. Lockwood et al., 1998; Pfaff et al., 1998). It may be that overlapping signatures and cusp ion steps are indeed the same phenomenon, viewed from middle and low altitudes respectively (see Lockwood and Smith, 1994; Lockwood, 1995; Trattner et al., 1998). That is an interesting, but entirely separate debate that has yet to be resolved. We simply wish to point out that by citing an example of overlapping signatures instead of one of the many clear examples of cusp ion steps (that we referred to), Heikkila is confusing the issue with a second and irrelevant debate.
It is not clear what point Heikkila is trying to make about the definition of reconnection. The implication is that our definition (of transfer of magnetic flux from open to closed) is different from Sonnerup's (of the presence of a tangential electric field in the magnetopause). Faraday's law states that these two things are uniquely the same thing.

Even for steady state, Heikkila provides an incorrect description of much of the closure of the dayside magnetopause currents and of the main sources of the Poynting flux which is deposited there [see Cowley (1991) for a correct description].

The current circuits are not incomplete in reconnection theory. But the reconnecting magnetosphere is not a single, simple circuit as Heikkila imagines. All currents are parts of loops and a close approximation current continuity is maintained. If Heikkila could show that reconnection theory (time-satationary or time-dependent) violates $\nabla \cdot \mathbf{J} \approx 0$ or $\nabla \cdot \mathbf{B} \approx 0$ that would be a valid objection - but he has not.

The concepts we apply are not two-dimensional. We do apply cylindrical symmetry along the reconnection lines for simplicity but, for example, our figures (that he reproduces) are inherently three dimensional in nature. Heikkila states "it is difficult to represent a threedimensional, time dependent situation with a steadystate two-dimensional model". We agree, although "impossible" would be a more accurate word than "difficult". That is why we used a three-dimensional, time-dependent model. By neglecting the $\partial W_{\mathrm{B}} / \partial \mathrm{t}$ term in Poynting's theorem and discussing only loads and dynamos, Heikkila is not using a time-dependent analogy.

Heikkila claims we contradict ourselves by illustrating, in Figs. $1 \mathrm{~b}$ and $1 \mathrm{c}$, cases where the ionospheric electric field $E_{\mathrm{i}}$ is zero. He does this only because he chooses to apply the Alfvén wave travel time of $1 \mathrm{~min}$ that we invoke on p. 868 to the "short time scales" we refer to on p. 867 , whereas we were referring to the 10 $15 \mathrm{~min}$ to establish full ionospheric flow. Immediately after the definition of the Alfvén transit time cited by Heikkila we state that the ionospheric flow (and therefore $E_{\mathrm{i}}$ ) is zero until the Alfvén wave arrives and then would increase over the subsequent 10-15 min. There is no contradiction here and we cannot understand how Heikkila has managed to make the wrong inference.

Heikkila makes unsupported statements like "they do not properly take account of the fact that the relevant processes operate in the presence of a plasma". Because there is no explanation, we do not know what he means by this. We note that it is not our argument that is based on analogies to toasters and batteries.

Heikkila states "Lockwood and Davis assume that the reconnection process is necessary to create a rotational discontinuity". This is not true. However, we do make use of the fact that the reconnection process leads to the production of a rotational discontinuity.

Lastly, Heikkila uses the comment to again put forward his rival theory to reconnection. This theory was rejected by most scientists because it used the 
equations of ideal MHD to produce a violation of ideal MHD, with no additional ingredient to cause that violation. Owen and Cowley (1991) showed that the missing ingredient was in fact an error in the way Heikkila had applied Faraday's law. Owen and Cowley's argument has not been refuted in the literature (Heikkila, 1992; Owen and Cowley, 1992). We do not think it necessary to comment further.

\section{References}

Cowley, S. W. H., Acceleration and heating of space plasmas: basic concepts, Annales Geophys., 9, 176, 1991.

Heikkila, W. J., Comment on Owen and Cowley's analysis of impulsive plasma transport through the magnetopause, $J$. Geophys. Res., 97, 1639, 1992.

Heikkila, W. J., Comment on Lockwood and Davis "On the longitudinal extent of magnetopause reconnection pulses", Ann. Geophys., 173-177, 1999.

Lockwood, M., Overlapping cusp ion injections: an explanation invoking magnetopause reconnection, Geophys. Res. Lett, 22, 1141-1144, 1995

Lockwood, M., and C. J. Davis, On the longitudinal extent of magnetopause reconnection bursts, Annales Geophys., 14, 865878, 1996.
Lockwood, M. and M. F. Smith, Low- and mid-altitude cusp particle signatures for general magnetopause reconnection rate variations: 1 theory, J. Geophys. Res., 99, 8531-8555, 1994.

Lockwood, M., C. J. Davis, T. G. Onsager, and J. A. Scudder, Modelling signatures of pulsed magnetopause reconnection in cusp ion dispersion signatures seen at middle altitudes, Geophys. Res. Lett., 25, 591-594, 1998.

Owen, C. J., and Cowley S. W. H., Heikkila's mechanism for impulsive transport through the magnetopause: a re-examination, J. Geophys. Res., 96, 5565-5574, 1991

Owen, C. J., and Cowley S. W. H., Reply to Heikkila's : "Comment on Owen and Cowley's analysis of impulsive plasma transport through the magnetopause", J. Geophys. Res., 97, 1641-1643, 1992.

Pfaff, R., J. Clemmons, C. Carlson, R. Ergun, J. McFadden, F. Mozer, M. Temerin, D. Klumpar, W. Peterson, E. Shelley, E. Moebius, L. Kistler, R. Strangeway, R. Elphic, and C. Cattell, Initial FAST observations of acceleration processes in the cusp, Geophys. Res. Lett., 25, 2037-2040, 1998.

Trattner, K. J., A. J. Coates, A. N. Fazakerley, A. D. Johnstone, H. Balsiger, J. L. Burch, S. A. Fuselier, W. K. Peterson, H. Rosenbauer, and E. G. Shelley, Overlapping ion populations in the cusp: POLAR/TIMAS results, Geophys. Res. Lett., 25, 1621-1624, 1998.

Woch J., and R. Lundin, Signatures of transient boundary layer processes observed with Viking, J. Geophys. Res., 97, 14311447, 1992. 